\title{
UDP-GLYCOSYLTRANSFERASES OF PLANT HORMONES
}

\author{
Maciej OSTROWSKI, Anna JAKUBOWSKA
}

\author{
Department of Biochemistry, Faculty of Biology and Environmental Protection, \\ Nicolaus Copernicus University in Torun
}

DOI: $10.2478 / \mathrm{acb}-2014-0003$

Summary: UDP-glycosyltransferases (GTases, UGT) catalyze the transfer of the sugar moiety from the uridine-diphosphate-activated monosaccharide (e.g. uridine-diphosphate-5'-glucose, UDPG) molecule to the specific acceptor. Glycosides contain aglycons attached by a $\beta$-glycosidic bond to $\mathrm{C} 1$ of the saccharide moiety. Glycosylation is one of the mechanisms maintaining cellular homeostasis through the regulation of the level, biological activity, and subcellular distribution of the glycosylated compounds. The glycosides play various functions in plant cells, such as high-energy donors, or signalling molecules, and are involved in biosynthesis of cell walls. Plant cells exhibit structural and functional diversity of UGT proteins. The Arabidopsis thaliana genome contains more than 100 genes encoding GTases, which belong to 91 families, and are deposited in the CAZY (Carbohydrate Active enzyme) database (www. cazy.org/GlycosylTransferases.html). The largest UGT1 class is divided into 14 subfamilies (A-N), and includes proteins containing highly conserved 44-amino acid PSPG (Plant Secondary Product Glycosyltransferase) motif at the C-terminus. The PSPG motif is involved in the binding of UDP-sugar donors to the enzyme. UGT1's catalyze the biosynthesis of both ester-type and ether-type conjugates of plant hormones (phytohormones). Conjugation of the phytohormones is an important mechanism that regulates the concentration of physiological active hormone levels during growth and development of plants. Glycoconjugation of phytohormones is widespread in the plant kingdom and all known phytohormones are able to form these conjugates. Most plant hormone conjugates do not indicate physiological activity, but rather are involved in transport, storage and degradation of the phytohormones. UDPG-dependent glycosyltransferases possess high substrate specificity, even within a given class of phytohormones. In many cases, the phenotype of plants is strongly affected by loss-of-function mutations in $U G T$ genes. In this paper, advances in the isolation and characterization of glycosyltransferases of all plant hormones: auxin, brassinosteroids, cytokinin, gibberellin, abscisic acid, jasmonates, and salicylate is described.

Key words: auxin, brassinosteroid, cytokinin, glycosylation, phytohormones, UDP-glycosyltransferases

Abbreviations: ABA - abscisic acid BL - brassinolide; BSMT - salicylic acid carboxyl methyltransferase; DON - deoxynivalenol; GA - giberellic acid; GT - glucosylotransferase; HK - histidine kinase; IAA - indole-3-acetic acid; IAGLU - IAA glucosyltransferase; JA - jasmonic acid; JGT - JA glucosyltransferase; Me - methyl salicylate; MeSAG - MeSA glucoside; SA - salicylic acid; SAGT/SGT - SA glucosyltransferase; TA - tuberonic acid; TAG - TA glucoside; UDPG - uridine-diphosphate-5' -glucose; ZOG - zeatin glucosyltransferase, ZOX - zeatin xylosyltransferase 


\section{INTRODUCTION}

Glycosylation, one of covalent modifications of low- and high molecular weight organic compounds forms an integral part of elementary and secondary metabolism, both in plants and in animal tissues [5, 26]. Attachment of a sugar moiety induces numerous alterations in physicochemical and biological properties of acceptor molecules, such as increases in their solubility and chemical stability, loss of reactivity or changes in biological activity.

Biosynthesis of glycosides for a long time was considered as a process of a restricted influence on plant physiology even if oligosaccharides and glycoconjugates and, in particular glycoproteins and glycolipids, intrigued biologists due to their unusual diversity and linked to it involvement as mediators in complex cellular processes. Glycosides play various functions in cells, participating in energy storage, formation of cell wall structure, signalling, and in intercellular or host-pathogen interactions $[2,3,48]$.

Biological effects of glycosylation in plant cells are particularly interesting in the case of the phytohormones, key regulators of plant growth and development. Physiological roles of phytohormones requires a precise control of their concentrations in the cell. Conjugation of plant hormones with sugars, amino acids or proteins represents one of the elements which maintain tissue homeostasis and occurs in the case of all plant hormone classes [1, 13,31]. Glycosyltransferases decrease the level of an active hormone through synthesis of hormone glycosides and esters. In most cases phytohormone conjugates display no activity as signalling molecules, but they fulfil other functions, such as being involved in hormone transport and storage and their degradation. Glycosylation of plant hormones is interesting because it is imf portant not only for phytohormone turnover, but also for their temporary inactivation. Conjugation, followed by hydrolysis of the bound forms, maintains hormone homeostasis without de novo synthesis of regulatory molecules $[1,26]$.

\section{GLYCOSYLOTRANSFERASES}

Glycosyltransferases may attach sugar moieties from molecules of active donors to substrates through $\mathrm{O}$ atoms of $-\mathrm{OH}$ and $-\mathrm{COOH}$ groups (the so called $O$-glycosylation), or also N, S and $\mathrm{C}$ atoms $[2,3]$. The sugar residue donor most frequently involves uridine-diphosphate-5'-glucose (UDP-glucose, UDPG) (fig. 1), but enzymes which use UDP-galactose, UDP-xylose, UDP-ramnose or UDP-glucuronate have also been identified. The process of $O$-glycosylation is conducted by uridine-diphosphosugar-dependent glycosyltransferases (UGT), ubiquitous enp zymes whose abundance matches the wide range of glycosides found in plants [6]. 


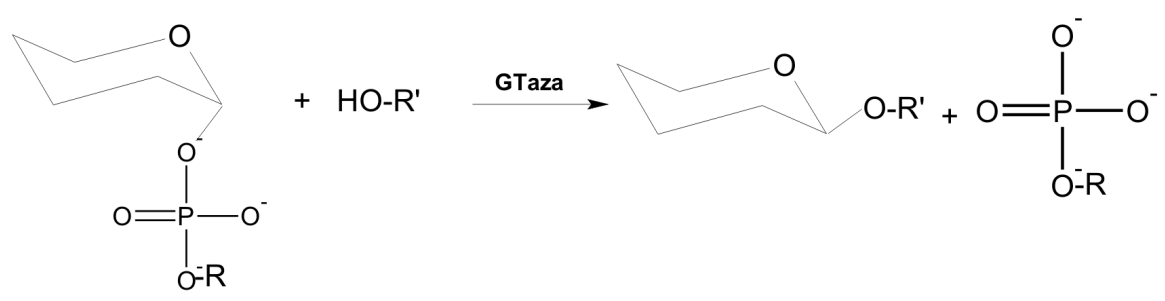

donor reszty cukrowcowej

glukozyd

(np UDPG)

FIGURE 1. Scheme of the reaction catalyzed by UDP-glucosyltransferases (based on [34], modified)

The characterisation and classification of glycosylotransferases is difficult bee cause of their structural and functional heterogeneity. For example, the genome of Arabidopsis thaliana contains over 100 genes for glycosyltransferases, belonging to 91 families, deposited in the CAZY (arbohydrate Active enzyme) database, which reveal diverse enzymatic activities toward acceptors of sugar residues [2, 3, 6, 35]. Based on phylogenetic analyses, UGT proteins were grouped into three classes [29]. The most numerous class of the enzymes, UGT1, contains 14 subclasses $(A-N)$, including enzymes participating in glycosylation of phytohormones and xenobiotics, while the two remaining less numerous classes include GTases, the substrates of which are sterols and other lipids.

The present paper describes enzymes classified as GTases of the first class (UGT1) which, as mentioned, catalyse phytohormone glycosylation [29, 50]. The structure of GTases contains a conserved PSPG (Plant Secondary Product Glycosyltransferase) motif of 44 amino acids (fig. 2), responsible for binding the highly energetic glucose donor, UDPG $[18,34]$. This motif is present at the $\mathrm{N}$-terminus and it participates in binding of a sugar residue donor.

Analysis of protein spatial structures, the sequences of which were deposited in the CAZY data base, demonstrated that the UGT1 enzymes contain the so called GT-B fold. Consistent with the model, the N-terminus of UGT1 protein is comprised of 7 parallel $\beta$ strands (N $\beta 1-8$ ), separated by eight $\alpha$-helical segments ( $\mathrm{N} \alpha 1-8$ ). The $\mathrm{C}$-terminus consists of six $\beta$-strands and eight $\alpha$-helices. The $\mathrm{N}$-terT minal fragments of GTases in this class is less conserved than their C-terminal fragments, which seems logical taking into account the fact that the $\mathrm{N}$-terminal participates in binding of various aglycons, i.e. acceptors of the sugar residue. It has been precisely determined which amino acid residues are involved in substrate binding. The acidic amino acids and glutamine situated in the loop N 33 and $\mathrm{N} \alpha 3$ can form hydrogen bonds with a glucose acceptor. The structure of $\mathrm{N} \beta 3 / \mathrm{N} \alpha 3$ subt strate-binding loop seems to be crucial for attachment of a saccharide acceptor. 


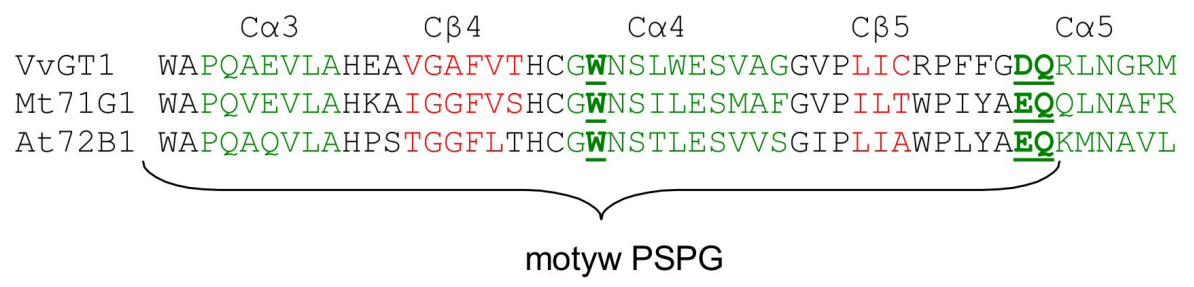

FIGURE 2. Alignment of PSPG motif sequences in three UDP-glucosyltransferases (VvGT1 - Vitis vinifera GT1, Mt71G1 - Medicago truncatula 71G1, At72B1 - Arabidopsis thaliana 72B1). $\alpha$-Helices in green and $\beta$-sheets in red. Three sugar interacting amino acid residues ( $\mathrm{W}$-tryptophan, $\mathrm{D} / \mathrm{E}$-asparh tate/glutamate, and Q-glutamine) are underlined in bold (based on: [34], modified)

Indeed, the enzyme UGT85H2 that binds a triterpene molecule containing a long ring structure, indicates a broad $\mathrm{N} \beta 3 / \mathrm{N} \alpha 3$ loop. However, the mechanism has not been unequivocally proven, since another related enzyme, UGT72B1, with a similar loop appears to be specific for such simple compounds as phenols or aniline.

The suggested mechanistic pathway of glycosyltransferase-catalysed reactions occurs in the following way: the residue of histidine 19/20, but probably also other conserved histidines, detach proton/s of the carboxy group of sugar acceptors, forming an oxyanion/s, which conduct a nucleophilic attack of $-\mathrm{OH}$ group by the C1 carbon of UDP-glucose [50]. The protonated histidine 19/20 is stabilized by a hydrogen bond formed with the aspartate 119 residue

\section{GLYCOSYLTRANSFERASES OF AUXINS}

Auxins represent the earliest recognised small group of phytohormones, among which indole-3-acetic acid (IAA) is the most important [12, 13, 20]. Free IAA, the biologically active form of the phytohormone, forms just a small fraction of the entire auxin pool in plants while auxin conjugates are the dominant form. Glycosyltransferases decrease levels of active auxin by catalysis of phytohormone ester synthesis. It should be emphasised that UGT1s are frequently involved only at the first stage of ester conjugate biosynthesis, forming a covalent bond between the carboxyl group in the hormone and the hydroxyl group at $\mathrm{C} 1$ in $\beta$-D- glucopyranose hemiacetal, e.g., 1-O-indole-3-acetyl $\beta$-D-glucose (fig. $3)$. Such a product represents a highly energetic unstable intermediate compound, providing a substrate in synthesis of the proper ester conjugates [20]. 


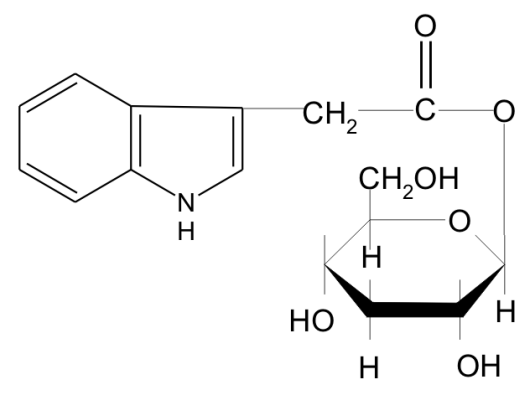

\section{1-O-indolilo-3-acetylo-ß-D-glukoza}

FIGURE 3. The structure of an $1-O$-indole-3-acetyl- $\beta$-D-glucose

IAA-glyco sydes are widespraead in plants, however their metabolism is relaf tively poorly recognised. UDPG-dependent IAA glycosyltransferase (EC 2.4.1.121) was isolated and purificated from endosperm of immature maize seeds. The enzyme catalyses synthesis of 1-O-indolyl-3-acetyl- $\beta$-D-glucose (1-O-IAGlc) in the reaction of glucose residue transfer to IAA carboxy group in the reaction of:

$$
\mathrm{IAA}+\mathrm{UDPG} \leftrightarrow 1-O-\mathrm{IAGl}+\mathrm{UDP}
$$

1-O-IAGlc synthase is a monomeric enzyme with a molecular weight of 46.5 $\mathrm{kDa}[13]$. The enzyme is very specific in respect to indole-3-acetic acid as an acceptor of the sugar residue and UDPG as the glucose donor. The -SH group of cysteine is essential for catalysis and regulation because tiols substantially increased enzyme activity. Development of maize seeds is linked to alterations in auxin levels, results ing from activity of UDP-glycosyltransferase. This is indicated by the fact that expression of the enzymatic protein is induced by a synthetic auxin, 1-naphthyl-acetic acid (1-NAA), and its activity is 14-fold higher in endosperm of immature seeds than in germinating seeds [15].

Polyclonal antibodies developed against 1-O-IAGlc synthase enabled the screening of a cDNA library from maize endosperm and identification of the ZmIAGLU gene which codes 1-O-IAGlc synthase [13, 15, 21]. In silico analyses demonstrated that this amino acid sequence of IAA glycosyltransferase contains at its C-terminus the PSPG motif, characteristic of class 1 UPDG transferases. It cannot be excluded that activity of the enzyme is controlled by covalent modifications, such as $\mathrm{N}$-gly- 
cosylation or $O$-phosphorylation, since the first order structure of ZmIAGLU was found to contain three sites for a potential $N$-glycosylation $(\mathrm{N}-\mathrm{X}(\mathrm{S}, \mathrm{T}) ; \mathrm{N}-\mathrm{S}, \mathrm{N}-\mathrm{T})$ and the $\mathrm{S} / \mathrm{T}-\mathrm{XR} / \mathrm{K}$ motif specific for substrates of protein kinase $\mathrm{C}$ [21]. The suggestion of control over activity of IAGlc synthase by phosphorylation/dephosphorylation seems even more plausible since the enzyme purified from maize seeds was activated by calcium ions and calmodulin.

Synthesis of IAGlc occurs universally since $I A G L U$ genes have also been identified also in other plants, such as Arabidopsis, duckweed, cauliflower, soyabean, tomato and tobacco plant $[12,13]$. Heterologic expression of maize 1-O-IAGlc synthase in tomato tissues suggests that the enzyme participates in control of IAA levels during plant growth and development $[9,21]$. Overexpression of ZmIAGLU reduces root development in tomato in parallel to a decrease in free auxin levels. Similar results were obtained in studies conducted on Arabidopsis thaliana [11]. Analysis of the phenotype in Arabidopsis overexpressing ZmIAGLU demonstrated that such transgenic plants revealed poor development of side radices and side leaves, which was linked to the lowered concentration of IAA as a result of an intense synthesis of IA-glucose. The growth inhibition could not be observed following action of IBA (indole-3-butyric acid) or 2,4-D (2,4-dichlorphenyloxyacetic acid), what pointed to specificity of the enzyme in respect to IAA. Overexpression of ZmIAGLU disturbed auxin equilibrium by reducing IAA level below the concentration which induced synthesis of IAA-aspartate and IAA-glutamate, the main conjugates of auxin in tissues of Arabidopsis. The high specificity of UGT, as related to auxins, was suggested also by the fact that the genome of the plant was found to contain the UGT74E2 gene, which codes for an IBA-specific glycosyltransferase [45]. Overexpression of the $U G T 74 E 2 O E$ gene resulted in phenotypic traits typical for overexpression of IAA glycosyltransferase and, in addition, it increased plant resistance to drought and salinity. Most probably, the genome of Arabidopsis thaliana contains more genes which code for IBA-glycosyltransferases, since a null mutation of UGT74E2 gene did not change the phenotype of the plant.

IAGlc synthase purified from immature seeds of pea (Pisum sativum L.) exhibits high enzymatic activity which is comparable to ZmIAGlu activity [14]. The significance of IAGlc synthesis in dicotyledonous plants is interesting since, as mentioned earlier, 1-O-indolyl-3-acetyl-glucose represents an unstable intermediate compound in the synthesis of the appropriate IAA esters. While enzymes catalysing transfer of an IAA residue from 1-O-IA-glucose to myo-inositol (1-O-IAGlc acyltransferase: myo-inositol), as well as in galactose-containing oligosaccharides (Sugar Acyltransferase, SugAc), are functional and present in maize endosperm [13] [43], until recently no acceptor of the indolylacyl residue from 1-O-IA-glucose had been been identified. The acceptor certainly does not involve amino acids since the mechanism of IAA amide conjugate synthesis proved to be entirely distinct [27]. 
Even if ester conjugates represent the main form of auxin in tissues of monocotyledonous plants, it is apparent from studies of the A. thaliana genome that four genes coding UDPG-indol-3-ylacetyl glycosyltransferase were present (UGT84B1, UGT84B2, UGT75B1, UGT75B2) [10]. Kinetic analyses have demonstrated that UGT84B1 glycosyltransferase has a clear preference for IAA as an acceptor of glucose. UDPG: indole-3-acetic acid glycosyltransferase from A. thaliana plays the key role in hormonal homeostasis, since overexpression of UGT84B1 in Arabidopsis results in an abrupt increase in IAA-glucose and IBA-glucose levels, manifested by insensitivity of the plants to biologically active auxin [11]. The normal phenotype becomes re-established following application of exogenous 2,4-D, the synthetic auxin, which is not a substrate in the reaction catalysed by UGT84B1.

\section{GLYCOSYLTRANSFERASES OF BRASSINOSTEROIDS}

Brassinosteroids, the plant steroid hormones, attach glucose residues through a hydroxyl group in position 23 (fig. 4) [38]. In tissues of Arabidopsis thaliana the modification is catalysed by the UDP-glycosyltransferase, UGT73C5 and its homologue, UGT73C6 [8, 32]. Apart from brassinolide (BL), the substrates for the recombined enzyme UGT73C5 include also castasterone and brassinosteroid precursors: tifasterole and 6-deoxycastasterone, and also a mycotoxin of the fungus Fusarium, deoxynivalenole (DON) [32]. Most probably, synthesis of brassinosteroid glycosides controls the concentration of the physiologically active phytohormone, since $U G T 73 C 5$ gene is expressed in hypocotyls and roots of young seedlings and levels of its mRNA decreases during plant development. Overexpression of UGT73C5 erases sensitivity of Arabidopsis tissues to brassinosteroids while silencing of the transcription results in an absence of $23-O$-glucoside BL synthesis. UGT73C6 and its homologue (UGT73C6) are expressed in the same tissues which are the sites of an intense synthesis of brassinosteroids. On the other hand, brassinolid does not induce UGT73C5 and UGT73C6 expression. Transcription of UGT73C6 is stimulated by DON, the herbicide of imidazoline, oligogalactouronides of plant cellular walls, salt stress and by infection with Botrytis cinerea [8]. This suggests involvement of both glycosyltransferases in, i.e., detoxication of xenobiotics, particularly because the UGT73C5 and UGT73C6 genes are located at branching sites of the phylogenetic tree corresponding to flavonoid GTases. An intriguing problem is posed by the intracellular localization of UGT73C5 and UGT73C6 in that they are present not only in the cytoplasm, but also in cell nucleus. This is striking because in contrast to animal steroid hormones no nuclear receptors have been identified for brassinosteroids. 


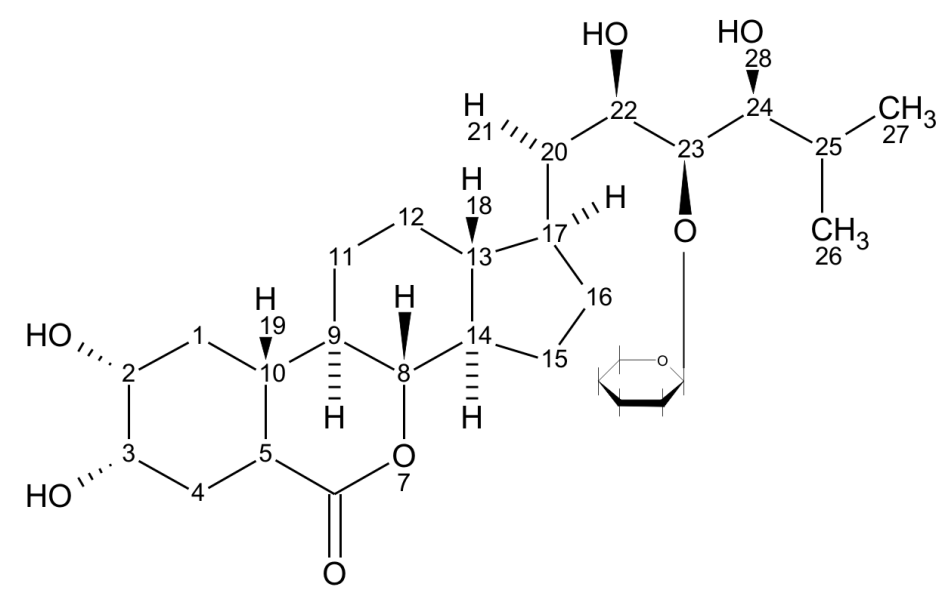

23-O-glukozyd brassinolidu

FIGURE 4. The structure of a/the brassinosteroid-glycoside

\section{GLYCOSYLTRANSFERASES OF CYTOKININS}

Physiological activity of cytokinins is demonstrated by purin nitrogen bases, nucleosides and their derivatives with an attached carbohydrate chain (isopentenyladenine) or with an aromatic ring (benzyladenine) [1]. Similar to other plant hormones, cytokinins form conjugates with sugars or amino acids. The presence of nitrogen atoms in cytokinin molecules causes them to form both $N$ - and $O$-glycosides (fig. 5). The main bound form of cytokinins involves conjugates with glucose $[1,12] . O$-glycosyltransferase specific to trans-zeatin (EC 2.4.1.203) was the first isolated cytokinin GTase, purified from immature seeds of Phaseolus lunatus [1]. The product of the enzyme-catalysed reaction, trans-zeatin $O$-glucoside, protects the phytohormone from oxydative degradation, as well as being its storage and transport form.

Analysis of expression products in libraries of cDNA clones from Phaseolus lunatus seeds permitted identification of the ZOG1 (Zeatin O-Glucosyltransferase 1) gene. In seeds of Phaseolus vulgaris L. expression of the ZOXI (Zeatin $\boldsymbol{O}$-Xylosyltransferase 1) gene takes place, which encodes for a UDP-xylosyltransferase of trans-zeatin (EC 2.4.2.40) [30]. The recombinant enzymatic protein reveals a high similarity to ZOG1.

Screening of genomic libraries of leguminous plants demonstrated other genes of potential cytokinin glycosyltransferases [23, 35]. Two genes in Phaseolus lunatus (PlGT2, PlGT3), one gene in Phaseolus vulgaris ( $P v G T 2)$ and two genes in Gly- 


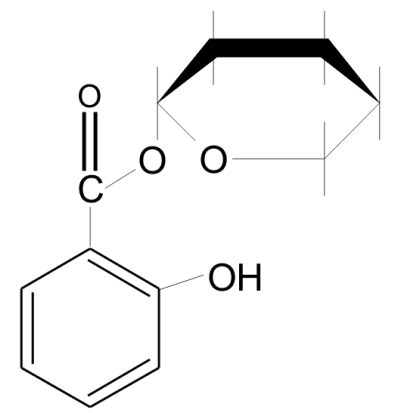

ester glukozowy kwasu salicylowego

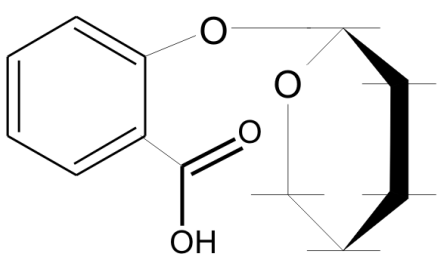

\section{O-glukozyd kwasu salicylowego}

FIGURE 5. The structures of $N$ - and $O$-cytokinin glycosides

cine $\max (G m G T 1, G m G T 2$ ) are highly homologous to ZOG1 and ZOX1. However, the function of proteins coded by the recognised genes remains unknown and none of them display catalytic activity of cytokinin GTase.

Cytokinin glycosyltransferases demonstrate substrate stereospecificity: they distinguish cis/trans isomers. There are two orthologs of $P$. lunatus ZOG1 in maize tissues, cisZOG1 and cisZOG2, which form $O$-glycosides of cis-zeatin [30]. The genes of cisZOG1 and cisZOG2 are $98.3 \%$ homologous to each other, but their expression pattern in tissues is different. Sensitivity of tissues to phytohormones seems to be reflected also in the process of their inactivation by conjugation. This thesis was confirmed by kinetic analyses, comparing binding of natural and syn- 
thetic cytokinins to receptors and the glycosyltransferases of ZOG1 and cisZOG1 [25]. In maize cells the prevailing form of an active cytokinin involves cis-zeatin, the physiological activity of which is manifested following its binding to ZmHK1 receptor. Interestingly, the receptor protein, similarly to cisZOG1, binds cis-zeatin and $o$-substituted synthetic cytokinins: $o$-topolin (hydroxy derivative of benzyladenine) and $o$-hydroxytidiazurone. In turn, trans-zeatin, which performs hormonal function in Arabidopsis, and also $m$-topolin, manifested a specific binding to the receptor kinases of AtHK4 and ZOG1, but could not serve as a substrate for cisZOG1, or ligands for ZmHK1. The results additionally point to a competition for the hormone molecule between receptor protein and a conjugating enzyme. Thus, cytokinin binding to either of the proteins leads to completely distinct physiological effects. As a receptor it mobilizes a signalling pathway, whereas as a glycosyltransferase it decreases tissue sensitivity to phytohormone. Results of this competition seems to depend on hormone concentration, corresponding to values of $K_{m}$ for the enzyme and $K_{d}$ for the receptor.

An important role of cytokinin $O$-glycosyltransferases in maintenance of hormonal homeostasis in maize tissues is shown by the fact that overexpression of the ZOG1 gene results in an abrupt decrease of cytokinin concentration and in phenotypic traits specific for an insufficient level of the hormones (lower apical meristems, thin shoots, sagittate leaves, well developed roots) [47].

The genome of $A$. thaliana contains two genes that encode cytokinin $N$-glycosyltransferases: UGT73C1 and UGT73C2, which specifically catalyze the transfer of a glucose moiety to $N^{7}, N^{9}, N^{6}$ atoms of isopentenyladenine and $N^{6}$-bensyladenine [7]. Genetic studies have demonstrated that the UGT73C2 isoenzyme is responsible for control of active cytokinins concentration since Arabidopsis mutants of $u g t 76 c 2$ manifest higher sensitivity to cytokinins and contain lower levels of $\mathrm{N}$-glycosides [49]. Moreover, overexpression of the gene induces phenotypic alterations typical for growth of the plants in conditions of a lowered cytokinin concentration.

\section{GLYCOSYLTRANSFERASES OF GIBBERELLINS}

Similar to other phytohormones, glycosylation of gibberellins provides one of mechanisms for control of their endogenous level. Even if production of gibberellin glycosides is ubiquitous, as they have been identified and isolated from tissues of multiple plants, knowledge of the GTases involved in the process is elusive [12, 22, 31]. $\mathrm{GA}_{3}$-specific UDP -glcosyltransferase was purified from maturing fruits of Phaseolus coccineus beans. Apart from 3-O-GA - glucoside, the enzyme also formed, with much lower potency, $\mathrm{GA}_{7}-$ and $\mathrm{GA}_{30}$-glucose conjugates. Moreover, synthesis of ester conjugates of $\mathrm{GA}_{7}$ and $\mathrm{GA}_{9}$ with glucose, was catalysed by UGT 
identified in the cytosol fraction of Lycopersicon peruvianum tomato cells. It cannot be excluded that low chemical stability of gibberellin conjugates as well as variability of the giberrellin isomers are the cause for poor identification of gibberellin GTases.

\section{GLYCOSYLTRANSFERASES OF ABSCISSIC ACID}

The mechanism warranting reversible inactivation of $\mathrm{ABA}$ involves its conjugation with glucose. Such conjugates may manifest characteristics of ester compounds in cases when the glucose residue is attached through a carboxy group at $\mathrm{C} 1$ of $\mathrm{ABA}$ or of ether compounds when the $\mathrm{C} 1^{\prime} \mathrm{OH}$ group is involved in the reaction $[19,24,31,52]$.

The gene encoding ABA-UGT in seedlings of adzuki beans (Vigna angularis) was cloned in 2002 [52]. The genome of this plant contains two copies of the UGT gene, the expression of which is activated by $50 \mu \mathrm{M} \mathrm{ABA}$ or plant damage and drought. In silico analysis of UGT amino acid sequences revealed homology levels of 30-44\% to other UDP-glucosyltransferases. Similar to IA-glucose synthase from maize, UGT from adzuki beans contains the typical motifs: the PSPG signature of UDP-glycosyltransferases, three sites for $N$-glycosylation (NRSK, NGSG, NSTL), the signal sequence directing the protein to peroxisomes and the coiled coil forming region. The recombinant purified protein exhibits $\mathrm{ABA}$ ester synthetic activity, but it does not form ether linkages with glucose. The most preferred substrate of glycosyltransferase from the adzuki beans is trans-(+)-ABA, but the enzyme glycosylates also (+)-S-ABA and (-)- $R$-ABA. Optimum $\mathrm{pH}$ of GTase ranges from 6.0 to 7.4, which contradicts the values determined for ABA glycosyltransferase in cell cultures of Macleaya microcarpa ( $\mathrm{pH}$ 5.0) and other identified GTases ( $\mathrm{pH}$ 5.2). The extensive diversity of the values may reflect the fact that the analyses were conducted on recombined proteins obtained in bacterial cells, which undoubtedly may affect folding conditions of the polypeptide.

Several sequences which encode UDP-glucosyltransferases were found in the genome of Arabidopsis thaliana [34]. Six of them (UGT84B1, UGT75B1, UGT84B2, UGT71B6, UGT75B2, UGT73B1) encode polypeptides which exhibit activity as $( \pm)$-ABA-glucose synthases. The highest catalytic activity was manifested by the UGT84B1 enzyme, but only UGT71B6 demonstrated enantioselectivity toward (+)-ABA [17]. Synthetic derivatives of ABA, PBI-413 and PBI-410 which contain a benzene ring attached to an ABA molecule were better acceptors of glucose residues. Another ABA structural analogue, PBI- 82, containing a triple bond between carbon atoms, was also a preferable substrate to (+)-ABA [17, 33]. Possibly, the triple bond and hydrophobic properties of the benzene ring provide higher stability of the bound substrate and a better exposure of the carboxy group during enzymatic glucosylation. 
Involvement of ABA glycosyltransferases in control of hormonal homeostasis, required for physiological processes in plants was recognised in part due to mutations of the UGT71B6 gene in Arabidopsis [34]. Elimination of the gene using knocko$u t$ techniques failed to affect the plant phenotype, which suggested that Arabidopsis contains other glycosyltransferases which decrease the concentration of free ABA. Overexpression of UGT71B6 increased levels of ABA esters. Interestingly, UGT71B6 functions in control of ABA levels during development of cotyledons but it exerts no effect on the course of classical ABA-controlled processes, such as movements of stomata or maturation of seeds. The results suggest that expression of genes coding for abcissic acid glycosyltransferases remains under the control of tissue-specific promoters, consistent also with the results of studies performed on adzuki beans [52].

\section{GLYCOSYLTRANSFERASES OF SALICYLIC ACID}

The regulatory role of salicylic acid (SA) in responses of plants to stress indicates that it involves an important signalling molecule, the concentration of which has to be precisely controlled. SA forms both methyl esters (MeSA) and ester-type (SGE) or glycoside-type (2-O-, 5-O- salicylic acid glycoside, SAG) conjugates with glucose (fig. 6) $[4,28]$. SA-methyl esters may also form glycosides, e.g. MeSAG, the methyl ester of salicylic acid 2-O- $\beta$-D-glycoside. The biological significance of SA-conjuj gates remains unclear, but some of them are assumed to increase polarity of salicylic acid, which permits their accumulation in vacuoles and protection against degradation. Thus, it seems highly probable that glycosides represent storage and transport forms of SA. Moreover, SA glycosides are compounds with a high-energy potential for transferring an acyl moiety and, therefore, they may be involved in biosynthesis of aromatic compounds.

In tobacco plants, the gene encoding SAGT (Salicylic Acid Glycosyltransferase) EC 2.4.1.35) was identified and this enzyme formed both esters and glycosides of salicylic acid [16]. SAGTase possesses high homology with other UDP-glycosyltransferases. However, the tobacco SA-UGT participates in synthesis of phenolic secondary metabolites, as indicated by results of analysis of its substrate specificity. 3-hydroxyand 4-hydroxybenzoic acid and trans-cinnamic acid are better glucose acceptors than SA. This suggests also that SAGT participates in conjugation of SA precursors, in this way controlling its concentration. SA is also a preferred substrate in the reaction catalysed by AtSGT1 (UGT74F2) from Arabidopsis [4]. The enzyme is almost 50\% homologous to SAGTase from tobacco plants and contains the 44-amino acid PSPG motif. All the UDP-GT regulate concentration of biotic stress mediators, and are induced by SA, MeSA or infection [16,37, 40]. In the context of these results it was interesting to learn that overexpression of the AtSGT1 gene resulted in a lower accumulation of SA and SAG and a reduced resistance to infection with Pseudomonas syringae [41]. 


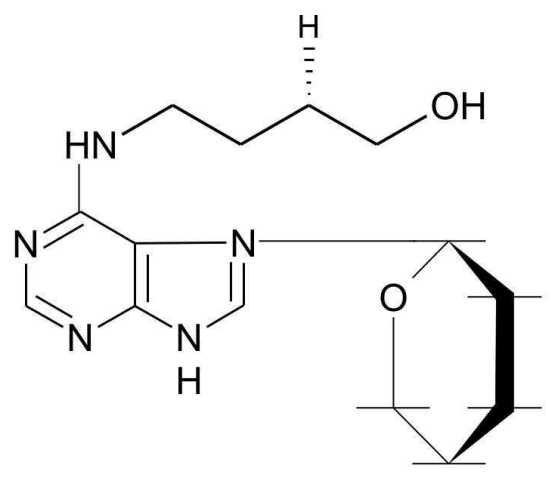

$N^{7}$-glukozyd dihydrozeatyny

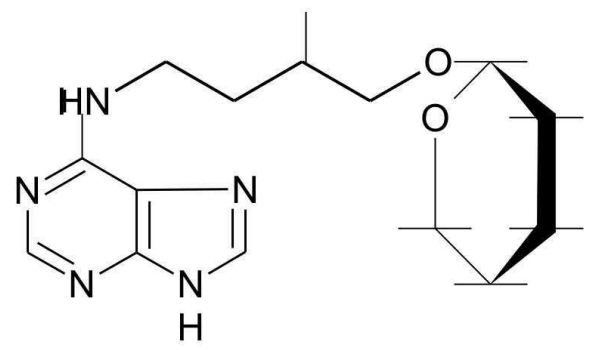

\section{O-glukozyd dihydrozeatyny}

FIGURE 6. The structures of a salicylic acid-glycoside and a glucose ester of salicylate

Attempting to explain these paradoxical results, the authors advanced a hypothesis assuming that high activity of AtSGT1, reflecting the overexpression, induced activity of salicylic acid methyltransferase, AtBSMT1 (Arabidopsis thaliana $\boldsymbol{S}$ A carboxyl Methyltransferase 1). This was supposed to lead to synthesis of methyl salicylate and MeSAG, particularly because SA manifests higher affinity to AtBSMT1 than to AtSGT1. The redundancy of proteins exhibiting activity of SA glycosyltransferases in tisa sues of A. thaliana was demonstrated by the fact that silencing of AtSAGT1 expression failed to affect the phenotype of the plants or SA and SAG levels [42]. Moreover, the genome of Arabidopsis contains as many as 14 genes coding for glycosyltransferases of hydroxybenzoate derivatives [16].

UDP-glycosyltransferase of SA in rice leaves is involved in systemic resistance of the plants since $O S S G T 1$, the gene coding for the enzyme was clearly induced by probenasole, the anti-mycotic chemical agent [46]. The purified, recombined 
OsSGT1 manifested high substrate specificity for salicylic acid and UDP-glucose. The rice reaction to probenasole was also specific since silencing of $O S S G T 1$ gene expression resulted in a lowered resistance in the plants to infection with Magnaporthe grisea fungus. These studies indicate that the SA glycoside represents the key molecule in the induction of a systemic plant protective response.

\section{GLYCOSYLTRANSFERASES OF JASMONIC ACID}

In contrast to other classical phytohormones, no specific jasmonic acid-GTase has been identified [51]. In 2005, Song reported that among six proteins homologous to AtSAGT only one, AtJGT1, manifested activity toward JA [39]. Nevertheless, detailed kinetic analyses performed using purified, recombinant AtJGT1 demonstrated that the glycosyltransferase preferred auxins, rather than JA.

Glycosylation of jasmonates was also linked to activity of SA GTases. An enzyme isolated from rice tissue cultures glycosylated both 12-hydroxy-epi-jasmonic acid (tuberonic acid, TA) and salicylic acid [36, 37]. Tuberonic acid represents a product of epi-jasmonic acid catabolism. An acceptor of a glucose moiety in the reaction catam lyzed by GTase may involve carboxyl or hydroxyl group, suggesting that SAGTase acts as an orthologue of UGT74F2 from Arabidopsis thaliana [16]. The product of the enzymatic reaction involves the tuberonic acid glycoside (TAG), the mobile signalling molecule, synthesised within plant responses to injury or to SA glycoside.

A NtSGT glycosyltransferase encoding gene was identified in tobacco plants, repg resenting an orthologue of SGT from rice [37]. The enzyme provides a good example that illustrates control of catalytic activity through accessibility of the substrate. Similar to OsSGT1, GTase from tobacco exhibits double activity toward TA and SA as the substrates in glycosylation. Following injury of the plant, NtSGT plays the role of a tuberonic acid UDP-glycosyltransferase, since the concentration of TA increases due to injury to the value of $K_{m}$ typical for the enzyme. On the other hand, salicylic acid acts as the glucose moiety acceptor in the reaction catalzyed by NtSGT during mycotic infection. Silencing of NtSGT gene expression using RNA interference reduces tobacco resistance to diseases induced by necrotrophic fungi.

\section{SUMMARY}

Plant hormone glycosylation is not only involved in the metabolism of signalling molecules, but also plays crucial roles in phytohormone homeostasis. Despite advances in identification and characterisation of plant glycosyltransferases, studies of glycosyltransferases in their functional context are far from easy ones due to the aforementioned extensive structural and functional variability of these enzymes. 
It should be noted that synthesis of phytohormone ester or ether conjugates could be also catalyzed by enzymes which are not specific for glucose acceptors. Immae ture seeds of Ipomoea nil were found to contain InGTase1, an enzyme that exhibits an equally high affinity to various phytohormones or their dervatives, including trans-ABA, IAA, SA, ( \pm )-JA, hydroxyderivatives of benzoic acid and cinnamic alcohol [44]. Interestingly, InGTase1 does not glucosylate gibberellins or transzeatine. The amino acid sequence of InGTase 1 resembles sequences of other glycoe syltransferases group 1, deposited in the CAZY database. InGTase1 activity could explain glycosylation of phytohormones in plants with loss-of-function mutations in UGT genes. The physiological significance of InGTase1 for maintenance of hormonal homeostasis remains still elusive. Synthesis of phytohormone-glycosides and phytohormone-glucose esters represents key elements affecting their physiologie cal concentrations. The significant role of UDPG-dependent glycosyltransferases is documented not only by the their multiplicity and variability, but also by their precise substrate specificity, even within the same phytohormone group. Moreover, in several cases mutations of GTase-coding genes strongly affect plant phenotypes. Earlier studies of UDPG-dependent glycosyltransferases were focused mainly on their isolation, purification and biochemical characterization, whereas contemporary studies, in view of advanced molecular biology techniques, allow for analysis of gene expression and for isolation of plant mutants with dysfunction of phytohormone glycosylation. To date, no promoter sequences of phytohormone GTase's have been described in detail. Indeed, elucidation of the molecular mechanism that regulates the expression of UGT genes will be a fascinating and revealing development.

\section{ACKNOWLEDGEMENTS}

The study was financed by the projects of MNiSW 0297/P01/2010/70 Iuventus Plus and ODW-7992/B/P01/2011/40 and by the UMK grant No. 1369-B

\section{REFERENCES}

[1] Bajguz A, Piotrowska A. Conjugates of auxin and cytokinin. Phytochem 2009; 70: 957-969.

[2] Bowles D, Isayenkova J, Lim E-K, Poppenberger B. Glycosyltransferases: managers of small molecules. Curr Opin Plant Biol 2005; 8: 254-263.

[3] Coutinho PM, Deleury E, Davies GJ, Henrissat B. An evolving hierarchical family classification for glycosyltransferases. J Mol Biol 2003; 328: 307-317.

[4] Dean JV, Delaney SP. Metabolism of salicylic acid in wild-type, ugt $74 f 1$ and ugt $74 f 2$ glucosyltransferase mutants of Arabidopsis thaliana. Physiol Plant 2008; 132: 417-425.

[5] Fedejko B, Mazerska Z. UDP-glycosyltransferases, ensoplasmic reticulum proteins - structure and mechanism of activity (in Polish). Post Biochem 2011; 57: 41-48.

[6] Halkjer Hansen E, Osmani Sa, Kristensen C, Lindberg Møller B, Hansen J. Substrate specificities of family 1 UGT gained by domain swapping. Phytochem 2009; 70: 473-482. 
[7] Hou B, Lim E-K, Higgins GS, Bowles DJ. $N$-glucosylation of cytokinins by glycosyltransferases of Arabidopsis thaliana. J Biol Chem 2004; 279: 47822-47832.

[8] Husar S, Berthiller F, Fujioka S, Rozhon W, Khan M, Kalaivanan F, Elias L, Higgins GS, Li Y, Schuhmacher R, Krska R, Seto H, Vaistij FE, Bowles D, Poppenberger B. Overexpression of the UGT73C6 alters brassinosteroid glucoside formation in Arabidopsis thaliana. BMC Plant Biol 2011; 11: 51 .

[9] Iyer M, Slovin JP, Epstein E, Cohen JD. Transgenic tomato plants with a modified ability to syntheh size indole-3-acetyl- $\beta$-1-O-D-glucose. J Plant Growth Regul 2005; 24: 142-152.

[10] Jackson RG, Lim E-K, Li Y, Kowalczyk M, Sandberg G, Hoggett J, Ashford DA, Bowles DJ. Identification and biochemical characterization of an Arabidopsis indole-3-acetic acid glucosyltransferase. J Biol Chem 2001; 276: 4350-4356.

[11] Jackson RG, Kowalczyk M, Li Y, Higgins G, Ross J, Sandberg G, Bowles DJ. Over-expression of an Arabidopsis gene encoding a glucosyltransferase of indole-3-acetic acid: phenotypic characterisation of transgenic lines. Plant J 2002; 32: 573-583.

[12] JaKuBowSKa A. Synthesis and hydrolysis of phytohormone conjugates in control of active hormone levels (in Polish). Post Biol Kom 2003; 30: 563-585.

[13] Jakubowska A. Mechanisms of IAA level control in plants (in Polish). Wydawnictwo UMK Torun, 2004.

[14] JakuBowska A, KowalczyK S. The auxin conjugat e1- $O$-indole-3-acetyl- $\beta$-D-glucose is synthesized in immature legume seeds by IAGlc synthase and may be used for modification of some high molecular weight compounds. J Exp Bot 2004; 55: 791-801.

[15] Kowalczyk S, Jakubowska A, Bandurski RS. 1-Naphtalene acetic acid induces indole-3-ylacetylglucose synthase in Zea mays seedling tissue. Plant Growth Regul 2002; 38: 127-134.

[16] Lim E-K, Doucet CJ, Li Y, Elias L, Worrall D, Spencer SP, Ross J, Bowles DJ. The activity of Arabidopsis glycosyltransferases toward salicylic acid, 4-hydroxybenzoic acid, and other benzoates. J Biol Chem 2002; 277: 586-592.

[17] Lim E-K, Doucet CJ, Hou B, Jackson RG, Abrams SR, Bowles DJ. Resolution of (+)-abscisic acid using an Arabidopsis glycosyltransferase. Tetrah: Assym 2005; 16: 143-147.

[18] Lorenc - KukuŁa K, Korobczak A, Aksamit - Stachurska A, Kostyń K, Łukaszewicz M, Szopa J. Glucosyltransferase: the gene arrangement and enzyme function. Cell Mol Biol Lett 2004; 9: 935-946.

[19] López-Carbonell M, Gabasa M, Jáuregui O. Enhanced det er minat ion of abscisic acid (ABA) and abscisic acid glucose ester (ABA-GE) in Citrus albidus plants by liquid chromatography - mass spectrometry in tandem mode. Plant Physiol Biochem 2009; 47: 256-261.

[20] Ludwig-Müller J. Auxin conjugates: their role for plant development and in the evolution of land plants. J Exp Bot 2011; 62: 1757-1773.

[21] Ludwig-Müller J, Walz A, Slovin JP, Epstein E, Cohen JD, Dong W, Town CD. Overexpression of maize IAGLU in Arabidopsis thaliana alters plant growth and sensitivity to IAA but not IBA and 2,4D. J Plant Growth Regul 2005; 24: 127-141.

[22] Marciniak K, Kęsy J, Tretyn A, Kopcewicz J. Gibberellins - structure, biosynthesis and inactivation in plants (in Polish). Post Biochem 2012; 58: 14-25.

[23] Meek L, Martin RC, Shan X, Karplus PA, Mok DWS, Mok MC. Isolation of legume glycosyltransferases and active site mapping of the Phaseolus lunatus zeatin O-glucosyltransferase ZOG1. J Plant Growth Regul 2008; 27: 192-201.

[24] Mimura A, Sumioka H, Matsunami K, Otsuka H. Conjugates of an abscisic acid derivative and phenolic glucosides, and a new sesquiterpene glucoside from Lindera strychnifolia. $J$ Nat Med 2010; 64: $153-160$.

[25] Mok MC, Martin Rc, Dobrev PI, Vanková R, Shing Ho P, Yonekura-Sakakibara K, Sakakibara $\mathrm{H}$, MoK DWS. Topolins and hydroxylated thidiazuron derivatives are substrates of cytokinin $O$-glucosyltransferase with position specificity related to receptor recognition. Plant Physiol 2005; 137: 1057-1066. 
[26] Osmani SA, BaK S, Lindberg Møller B. Substrate specificity of plant UDP-dependent glycosyltransferases predicted from crystal structures and homology modeling. Phytochem 2009; 70: 325-347.

[27] Ostrowski M, JaKuBowsKa A. Identification of enzyme activity that conjugates indole-3-acetic acid to aspartate in immature seeds of pea (Pisum sativum L.) J Plant Physiol 2008; 165: 564-569.

[28] Pastor V, Vincent C, Cerezo M, Mauch-Mani B, Dean J, Flors V. Detection, characterization and quantification of salicylic acid conjugates in plant extracts by ESI tandem mass spectrometric techniques. Plant Physiol Biochem 2012; 53: 19-26.

[29] Paquette S, Lindberg Møller B, BaK S. On the origin of family 1 plant glycosyltransferases. Phytochem 2003; 62: 399-413.

[30] Pineda Rodo A, Brugière N, Vankova R, Malbeck J, Olson JM, Haines SC, Martin RC, Habben JE, Мок DWS, Мок MC. Over-expression of a zeatin $O$-glucosylation gene in maize leads to growth retardation and tasselseed formation. $J$ Exp Bot 2008; 59: 2673-2686.

[31] Piotrowska A, Bajguz A. Conjugates of abscisic acid, brassinosteroids, ethylene, gibberellins, and jasmonates. Phytochem 2011; 72: 2097-2112.

[32] Poppenberger B, Fujioka S, Soeno K, George Gl, Vaistij Fe, Hiranuma S, Seto H, Takatsuto S, Adam G, Yoshida S, Bowles D. The UGT73C5 of Arabidopsis thaliana glucosylates brassinosteroids. Proc Natl Acad Sci USA 2005; 102: 15253-15258.

[33] Priest DM, Jackson RG, Ashford DA, Abrams SR, Bowles DJ. The use of abscisic acid analogues to analyse the substrate selectivity of UGT71B6, a UDP-glycosyltransferase of Arabidopsis thaliana. FEBS Lett 2005; 579: 4454-4458.

[34] Priest DM, Ambrose SJ, Vaistij FE, Elias L, Higgins GS, Ross ARS, Abrams SR, Bowles DJ. Use of the glucosyltransferase UGT71B6 to disturb abscisic acid homeostasis in Arabidopsis thaliana. Plant J 2006; 46: 492-502.

[35] Sado P-E, Tessier D, Vasseur M, Elmorjani K, Guillon F, Saulnier L. Integrating genes and phenotype: a wheat - Arabidopsis-rice glycosyltransferase database for candidate gene analyses. Funct Integr Genom 2009; 9: 43-58.

[36] Seto Y, Hamada S, Matsuura H, Matsushige M, Satou C, Takahashi K, Masuta C, Ito H, Matsui $\mathrm{H}$, NABETA K. Purification and cDNA cloning of a wound inducible glucosyltransferase active toward 12-hydroxy jasmonic acid. Phytochem 2009; 70: 370-379.

[37] Seto Y, Hamada S, Ito H, Masuta C, Matsui H, Nabeta K, Matsuura H. Tobacco salicylic acid glucosyltransferase id active toward tuberonic acid (12-hydroxyjasmonic acid) and is induced by mechanical wounding stress. Biosci Biotech Biochem 2011; 75: 2316-2320.

[38] Soeno K, Fujioka S, Hiranuma S, Seto H, Yoshida S. Metabolic conversion of castasterone and brassinolide into their glucosides in higher plants. J Plant Growth Regul 2006; 25: 195-202.

[39] Song JT. Biochemical charact er ization of an Arabidopsis glucosyltransferase with high activity toward jasmonic acid. J Plant Biol 2005; 48: 422-428.

[40] Song JT. Induction of a salicylic acid glucosyltransferase, AtSGT1, is an early disease response in Arabidopsis thaliana. Mol Cell 2006; 22: 233-238.

[41] Song JT, Koo YJ, Seo HS, Kim MC, Chог YD, Kim JH. Overexpression of AtSGT1, an Arabidopsis salicylic acid glucosyltransferase, leads to increased susceptibility to Pseudomonas syringae. Phytochem 2008; 69: 1128-1134.

[42] Song JT, Koo YJ, Park J-B, Seo YJ, Cho Y-J, Seo HS, Choi YD. The expression patterns of AtBSMT1 and AtSAGT1 encoding a salicylic acid (SA) methyltransferase and a SA glucosyltransferase, respectively, in Arabidopsis plants with altered defense responses. Mol Cell 2009; 28: 105-109.

[43] Starzyńska E, KowalczyK S. Novel 1-O-indole-3-acetyl- $\beta$-D-glucose-dependent acyltransferase transferring indoleacetyl moiety to some mono-, di, and oligosaccharides. Acta Physiol Plant 2012; 34: 53-63.

[44] Suzuki H, Hayase H, Nakayama A, Yamaguchi I, Asami T, Nakajima M. Identification and characterization of an Ipomoea il glucosyltransferase which metabolizes some phytohormones. Biochem Biophys Res Commun 2007; 361: 980-986. 
[45] Tognetti VB, Van Aken O, Morreel K, Vandenbroucke K, Van De Cotte B, De Clerq I, Chiwocha S, Fenske R, Prinsen E, Boerjan W, Genty B, Stubbs KA, Inzé D, Van Breusegem F. Perturbation of indole-3-butyric acid homeostasis by the UDP-glucosyltransferase UGT74E2 modulates Arabidopsis architecture and water stress tolerance. Plant Cell 2010; 22: 2660-2679.

[46] Umemura K, Satou J, Ifata M, Uozumi N, Koga J, Kawano T, Koshiba T, Anzai H, Mitomi M. Contribution of salicylic acid glucosyltransferase, OsSGT1, to chemically induced disease resistance in rice plants. Plant $J$ 2009; 57: 463-472.

[47] Veach YK, Martin RC, Mok DWS, Malbeck J, Vankova R, Mok MC. O-glucosylation of cis-zeatin in maize. Characterization of genes, enzymes, and endogenous cytokinins. Plant Physiol 2003; 131: 1374-1380.

[48] Wang J, Hou B. Glycosyltransferases: key players involved in the modification of plant secondary metabolites. Front Biol Chin 2009; 4: 39-46.

[49] Wang J, Ma X-M, Kojima M, Sakakibara H, Hou B-K. N-glucosyltransferase UGT76C2 is involved in cytokinin homeostasis and cytokinin response in Arabidopsis thaliana. Plant Cell Physiol 2011; 52: $2200-2213$.

[50] WANG X. Structure, mechanism and engineering of plant natural product glycosyltransferases. FEBS Lett 2009; 583: 3303-3309.

[51] Wilmowicz E, Frankowski K, SidŁowska M, Kućko A, Kęsy J, Gąsiorowski A, Glazińska P, KopcewicZ J. Jasmonate biosynthesis - the latest discoveries (in Polish) Post Biochem 2012; 58: 26-33.

[52] Xu Z-J, Nakajima M, Suzuki Y, Yamaguchi I. Cloning and characterization of the abscisic acid-specific glucosyltransferase gene from adzuki bean seedlings. Plant Physiol 2002; 129: 1285-1295.

Editor-Andrzej Kononowicz

Maciej Ostrowski

Department of Biochemistry

Faculty of Biology and Environmental Protection Nicolaus Copernicus University in Toruń

7 Gagarina Street

87-100 Toruń

tel.: 566114542

fax: 566114772

e-mail:maciejost@umk.pl 